УДК 517.75

Тор Клиффорда и бутылка Клейна

M.А. Чешкова

Алтайский государственный университет (Барнаул, Россия)

\title{
Clifford Torus and Klein Bottle
}

M.A. Cheshkova

Altai State University (Barnaul, Russia)

Если вдоль некоторой замкнутой кривой на поверхности локальная ориентация в касательном пространстве меняет знак, то поверхность называется односторонней. Простейшей односторонней поверхностью является лист Мёбиуса. К односторонним поверхностям относится также бутылка Клейна, скрещенный колпак. Бутылку Клейна можно рассматривать как два листа Мёбиуса, склеенные по краю.

В работе бутылка Клейна разрезается на два листа Мёбиуса.

Пусть на торе Клиффорда в $E^{4}$ задана замкнутая кривая с помощью $4 \pi$-периодической векторфункции. Используя найденную функцию, определяются уравнения листов Мёбиуса, бутылки Клейна. Если средняя линия одного из листов Мёбиуса вырождается в точку, то получим скрещенный колпак. С помощью системы компьютерной математики строятся индикатрисы нормальной кривизны исследуемых поверхностей. В случае бутылки Клейна индикатриса нормальной кривизны есть эллипс. Если бутылка Клейна вырождается в скрещенный колак, то индикатриса нормальной кривизны есть окружность.

Ключевые слова: бутылка Клейна, лист Мёбиуса, скрещенный колпак, $4 \pi$-периодическая функция.

\section{DOI 10.14258/izvasu(2017)1-29}

Если на поверхности в $E^{n}$ существует замкнутая кривая (дезориентирующий контур), обладающая тем свойством, что при ее обходе локальная ориентация в касательном пространстве меняет знак, то поверхность называется односторонней.

Впервые уравнение односторонней поверхности в $E^{3}$, открытой Мёбиусом, было получено Машке [1]. Если гауссова кривизна листа Мёбиуса равна нулю, то он называется плоским. Библиография работ на эту тему дана в работе [2].

K односторонним поверхностям относятся: скрещенный колпак [3], бутылка Клейна [3-5]. В [6] исследуется плоский лист Мёбиуса. Кроме того, односторонние поверхности и их приложения рассматриваются в работах [7-14].
A surface is called the one-sided surface if a local orientation along a closed curve on a tangent space changes its sign. The simplest one-sided surface is the Mobius strip. The Klein bottle and cross-cap are also one-sided surfaces. The Klein bottle can be considered as two Mobius strips glued on edges.

In this paper, the Klein bottle is cut into two Mobius strips. We define the closed curve on the Clifford torus in $E^{4}$ using $4 \pi$-periodic vectorfunction. We get a cross-cap if the Mobius strip degenerates into a point. Indicatrices of normal curvature for the investigated surfaces are studied with the help of computer mathematics. For the Klein bottle, the indicatrix of normal curvature is an ellipse. If the Klein bottle degenerates into a crosscap, then the indicatrix of normal curvature is a circle.

Key words: Klein bottle, Mobius band, cross-cap, $4 \pi$-periodic function.

В евклидовом пространстве рассмотрим гладкую замкнутую неплоскую кривую $\gamma$ без самопересечения, заданную $4 \pi$-периодической векторфункцией $\rho=\rho(v)$, которая не является $2 \pi$ периодической и $2 \pi$-антипериодической.

Так как $\rho(v)=\rho(v+4 \pi)$, то функция

$$
s(v)=\frac{1}{2}(\rho(v)+\rho(v+2 \pi)),
$$

есть $2 \pi$-периодическая не равная нулю, а векторфункция

$$
l(v)=\frac{1}{2}(\rho(v)-\rho(v+2 \pi))
$$

есть $2 \pi$-антипериодическая, не равная нулю. 
Используем найденные функции для построения модели бутылки Клейна.

Рассмотрим поверхность $K L$ :

$$
\begin{aligned}
& r(u, v)=(p+\cos (u)) s(v)+\sin (u) l(v), p \neq \mp 1, \\
& u \in[-\pi, \pi], v \in[-\pi, \pi] .
\end{aligned}
$$

Теорема 1. Поверхность KL односторонняя.

Доказательство. Рассмотрим замкнутую кривую (дезориентирующий контур) $r(0, v)=(p+1) s(v)$. Имеем $r_{1}=\left.r_{u}\right|_{u=0}=$ $l(v), r_{2}=\left.r_{v}\right|_{u=0}=(p+1) s^{\prime}(v)$.

Касательное пространство в точках кривой $S: r(0, v)=(p+1) s(v)$ примет вид

$$
T_{k} K L=\left\{l(v), s^{\prime}(v)\right\}, k \subset S
$$

Замечаем, что базисы

$$
\begin{gathered}
\left.\left\{r_{1}(v), r_{2}(v)\right\}=\{l(v)), s^{\prime}(v)\right\}, \\
\left\{r_{1}(v+2 \pi), r_{2}(v+2 \pi)\right\}=\left\{l(v+2 \pi), s^{\prime}(v+2 \pi)\right\}
\end{gathered}
$$

противоположно ориентированы. Поверхность односторонняя.

Нетрудно проверить, что и вдоль замкнутой кривой $S^{*}: r(\pi, v)=(p-1) s(v)$ локальные базисы также меняют ориентацию.

\section{Теорема 2.}

Поверхность $K L$ определяет модель бутылки Клейна.

Доказательство. Рассмотрим бутылку Клейна как фактор-пространство [15, с. 75$]$ :

$K^{*}=[-\pi, \pi] \times[-\pi, \pi] /[(-\pi, v) \sim$ $(\pi, v),(-u,-\pi) \sim(u, \pi)]$.

Для поверхности (3) имеем $r(-\pi, v)=(p-$ 1) $s(v)=r(\pi, v)$, что соответствует «склейке» $(-\pi, v) \sim(\pi, v)$

А так как $r(-u,-\pi)=(p+\cos (-u)) s(-\pi)+$ $\sin (-u) l(-\pi)=(p+\cos (-u)) s(\pi)-\sin (-u) l(\pi)=$ $r(u, \pi)$, то имеем «склейку» $(-u,-\pi) \sim(u, \pi)]$.

Поверхность Клейна можно получить, «склеив» два листа Мёбиуса по краю.

Рассмотрим кривую $r=r\left(u_{0}, v\right), v \in[-2 \pi, 2 \pi]$ на $K L$ и разрежем $K L$ вдоль нее. ный)

Получили лист Мёбиуса $K L 1$ (криволиней-

$$
r(u, v)=(p+\cos (u)) s(v)+\sin (u) l(v),
$$

$u \in\left[-u_{0}, u_{0}\right], v \in[-\pi, \pi]$.

Для листа $K L 1$ средней линией является линия $S: r(0, v)=(p+1) s(v)$, а краем - кривая $r=r\left(u_{0}, v\right), v \in[-2 \pi, 2 \pi]$.

Второй лист $K L 2$ задается уравнением

$$
r(u, v)=(p+\cos (u)) s(v)+\sin (u) l(v),
$$

$u \in\left[u_{0}, 2 \pi-u_{0}\right], v \in[-\pi, \pi]$.

Для листа $K L 2$ средней линией является линия $S^{*}: r(\pi, v)=(p-1) s(v)$. Край листа $K L 1$ совпадает с краем листа $K L 2$. $E^{4}$.

Будем строить рассматриваемые поверхности

Рассмотрим тор Клиффорда

$$
r(u, v)=(\cos (u), \sin (u), \cos (v), \sin (v)),
$$

$u \in[0,2 \pi], v \in[0,2 \pi]$.

Положим $u=v / 2$. Тогда

$$
\begin{gathered}
\rho(v)=\left(\cos \left(\frac{v}{2}\right), \sin \left(\frac{v}{2}\right), \cos (v), \sin (v)\right), \\
s(v)=(0,0, \cos (v), \sin (v)), \\
l(v)=\left(\cos \left(\frac{v}{2}\right), \sin \left(\frac{v}{2}\right), 0,0\right) .
\end{gathered}
$$

Имеем, используя (7),

$$
\begin{gathered}
r_{1}=r_{u}=-\sin (u) s(v)+\cos (u) l(v), \\
r_{2}=r_{v}=(p+\cos (u)) s^{\prime}(v)+\sin (u) l^{\prime}(v) .
\end{gathered}
$$

Определим метрический тензор $g_{i j}=\left(r_{i}, r_{j}\right)$ бутылки Клейна $K L$.

$$
g_{11}=1, g_{12}=0, g_{22}=\frac{1}{4} \sin (u)^{2}+(p+\cos (u))^{2} .
$$

Определим единичные нормальные векторы $n_{1}, n_{2}$ в произвольной точке поверхности $K L$.

$$
\begin{gathered}
n_{1}=\cos (u) s(v)+\sin (u) l(v), \\
n_{2}=\frac{1}{\sqrt{4 g_{22}}}\left(4(p+\cos (u)) l^{\prime}(v)-\sin (u) s^{\prime}(v)\right) .
\end{gathered}
$$

Пусть $t=t^{i} r_{i} \in T_{k} K L, k \in K L, r_{1}=r_{u}, r_{2}=$ $r_{v}$ - касательный вектор, длина которого равна единице. Рассмотрим вектор нормальной кривизны $b=b(t, t)$, где $b(t, t)$ - вторая фундаментальная форма поверхности $K L$.

Зафиксируем точку $k$, а вектор $t$ будем менять. Тогда концы вектора нормальной кривизны с началом в $k$ опишут в $T_{k} K L^{\perp}$ кривую, называемую индикатрисой нормальной кривизны [16, с. 253]. Индикатриса нормальной кривизны есть эллипс либо отрезок прямой.

Определим ее.

Имеем

$$
\begin{gathered}
t=\cos (\beta) \frac{r_{1}}{\left|r_{1}\right|}+\sin (\beta) \frac{r_{2}}{\left|r_{2}\right|}, \\
b(t, t)=\left(\cos (\beta)^{2} b_{11}^{1}+2 \sin (\beta) \cos (\beta) b_{12}^{1} \frac{1}{\sqrt{g_{22}}}+\right. \\
\left.\sin (\beta)^{2} b_{22}^{1} \frac{1}{g_{22}}\right) n_{1}+\left(\cos (\beta)^{2} b_{11}^{2}+\right. \\
\left.2 \sin (\beta) \cos (\beta) b_{12}^{2} \frac{1}{\sqrt{g_{22}}}+\sin (\beta)^{2} b_{22}^{2} \frac{1}{g_{22}}\right) n_{2}, \\
b_{i j}^{1}=<r_{i j}, n_{1}>, b_{i j}^{2}=<r_{i j}, n_{2}>
\end{gathered}
$$




$$
\begin{gathered}
b_{11}^{1}=-1, b_{11}^{2}=0, b_{12}^{1}=0, b_{12}^{2}=\frac{1+p \cos (u)}{\sqrt{4 g_{22}}}, \\
b_{22}^{1}=p(p+\cos (u))-g_{22}, b_{22}^{2}=0 .
\end{gathered}
$$

Итак,

$$
\begin{gathered}
b(t, t)=b^{1}(t, t) n_{1}+b^{2}(t, t) n_{2}, \\
b^{1}=-1+\frac{p(p+\cos (u))}{g_{22}} \sin (\beta)^{2}, b^{2}= \\
=\frac{p \cos (u)+1}{2 g_{22}} \sin (2 \beta) .
\end{gathered}
$$

Переходя к двойному углу, получим

$$
\begin{gathered}
b^{1}=-1+\frac{p(p+\cos (u)}{2 g_{22}}-\frac{p(p+\cos (u))}{2 g_{22}} \cos (2 \beta), \\
b^{2}=\frac{p \cos (u)+1}{2 g_{22}} \sin (2 \beta),
\end{gathered}
$$

Таким образом, имеет место следующая теорема.

Теорема 3. Индикатриса бутылки Клейна $K L$ при $p \neq 0$ есть эллипс с осями

$a_{1}=-\frac{p(p+\cos (u))}{2 g}, a_{2}=\frac{p \cos (u)+1}{2 g}, g=\operatorname{det}\left(g_{i j}\right)$.

При $p=0$ это отрезок прямой.

Построим индикатрисы нормальной кривизны при $p=2$ вдоль линий : $u=0$ (рис. 1 ) и $u=3 / 4 \pi$ (рис. 2).

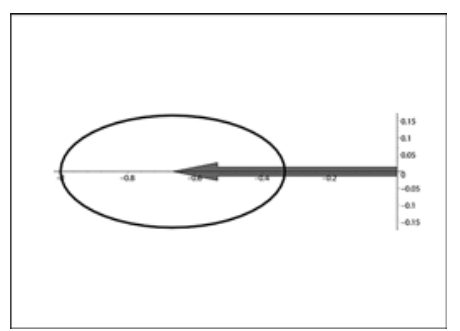

Рис. 1. Индикатриса нормальной кривизны и нормаль средней кривизны при $p=2, u=0$

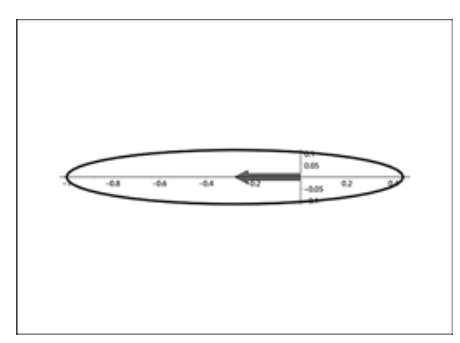

Рис. 2. Индикатриса нормальной кривизны и нормаль средней кривизны при $p=2, u=3 / 4 \pi$

Определим две матрицы $A_{1}, A_{2}[17$, с. 22]

$$
<A_{1}\left(r_{i}\right), r_{j}>=<b\left(r_{i}, r_{j}\right), n_{1}>,
$$

$$
<A_{2}\left(r_{i}\right), r_{j}>=<b\left(r_{i}, r_{j}\right), n_{2}>.
$$

Обозначим

$$
A_{\alpha}\left(r_{i}\right)=A_{\alpha i}^{k} r_{k}, \alpha=1,2, A_{\alpha i}^{k} g_{k j}=b_{i j}^{\alpha} .
$$

В силу (8), (9) получим

$$
\begin{aligned}
& A_{1}=\left(\begin{array}{cc}
-1 & 0 \\
0 & \frac{p(p+\cos (u))}{g}-1
\end{array}\right), \\
& A_{2}=\left(\begin{array}{cc}
0 & \frac{1+p \cos (u)}{2 g \sqrt{g}} \\
\frac{1+p \cos (u)}{2 g \sqrt{g}} & 0
\end{array}\right),
\end{aligned}
$$

$g=\operatorname{det}\left(g_{i j}\right)=g_{22}$.

Вектор $\eta=\frac{1}{2}\left(\left(\right.\right.$ trance $\left.A_{1}\right) n_{1}+\left(\right.$ trance $\left.\left.A_{2}\right) n_{2}\right)$ называется нормалью средней кривизны [17, с. 40].

Имеем $\eta=\left(\frac{p(p+\cos (u))}{2 g}-1\right) n_{1}$. Средняя кривизна $H$ равна $H=|\eta|=\left|\frac{p(p+\cos (u))}{2 g}-1\right|$.

Определим гауссову кривизну $K$ бутылки Клейна $K L[18$, c. 133$]$

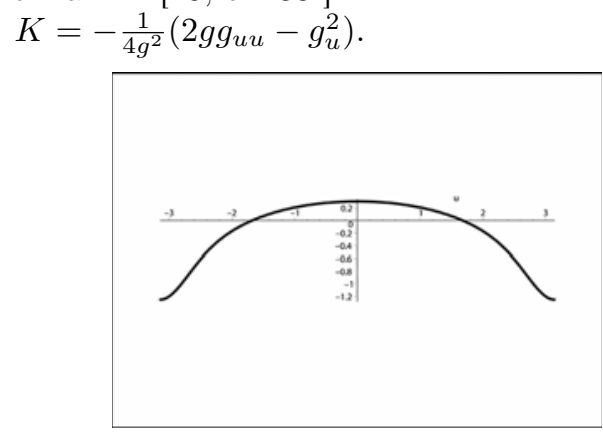

Рис. 3. Гауссова кривизна при $p=2, u \in[-\pi, \pi]$

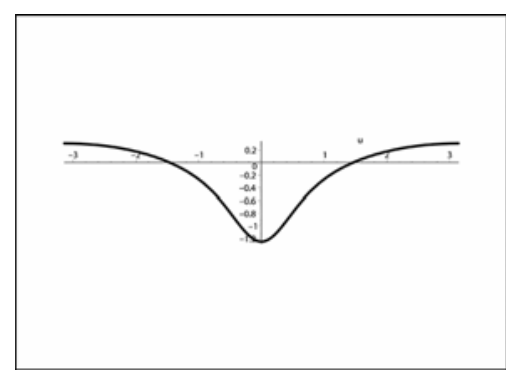

Рис. 4. Гауссова кривизна при $p=-2, u \in[-\pi, \pi]$

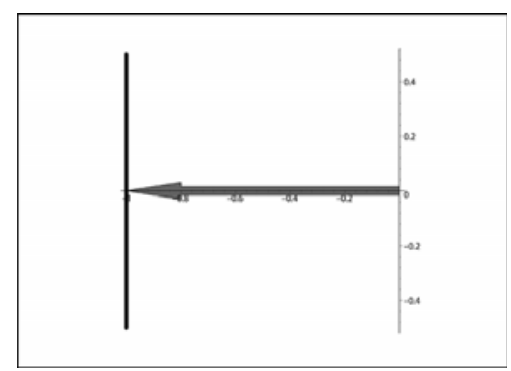

Рис. 5. Индикатриса нормальной кривизны и нормаль средней кривизны при $p=0, u=\pi / 4$ 
Используя математический пакет, построим графики (рис. 3-5). Остановимся подробнее на случае $p=0$. Имеем $\langle r, r\rangle=1, H=1$.

Теорема 4. Если $p=0$, mo

1) бутылка Клейна $K L$ принадлежит 3-сфере,

2) индикатриса нормальной кривизны есть отрезок прямой,

3) средняя кривизна $H=1$.

Лист Мёбиуса $K L 1$ задается уравнением

$$
\begin{gathered}
r(u, v)=(p+\cos (u))(0,0, \cos (v), \sin (v))+ \\
\sin (u)(\cos (v / 2), \sin (v / 2), 0,0),
\end{gathered}
$$

$u \in\left[-u_{0}, u_{0}\right], v \in[-\pi, \pi]$.
Для листа $K L 1$ средней линией является линия

$$
S: r(0, v)=(p+1)(0,0, \cos (v), \sin (v)) .
$$

Уравнение второго листа $K L 2$ имеет вид

$$
\begin{gathered}
r(u, v)=(p+\cos (u))(0,0, \cos (v), \sin (v))+ \\
\sin (u)(\cos (v / 2), \sin (v / 2), 0,0),
\end{gathered}
$$

$u \in\left[u_{0}, 2 \pi-u_{0}\right], v \in[-\pi, \pi]$.

Для листа $K L 2$ средней линией является линия

$$
S^{*}: r(\pi, v)=(p-1)(0,0, \cos (v), \sin (v)) .
$$

\section{Библиографический список}

1. Mashke H. Note on the unilateral surface of Moebius // Trans. Amer. Math. Soc., 1900. Vol. 1, № 1 .

2. Сабитов И.Х. Изометрические погружения и вложения плоского листа Мёбиуса в евклидовы пространства // Известия РАН. - 2007. Т. 71 , № 5 .

3. Кривошапко С.Н., Иванов В.Н., Халаби С.М. Аналитические поверхности. - М., 2006.

4. Гильберт Д., Кон-Фоссен С. Наглядная геометрия. - М., 1981.

5. Чешкова М. А. О бутылке Клейна // Известия Алтайского гос. ун-та. - 2012. - Т. 73, № 1/1.

6. Чешкова М. А. О плоском листе Мёбиуса // Известия Алтайского гос. ун-та. - 2013. - Т. 77, № $1 / 2$.

7. Борисюк А.Р. Глобальные бифуркации на бутылке Клейна. Общий случай // Математический сборник. - 2005. - Т. 196, № 4.

8. Набеева Л.Р. Классификация узлов в утолшенной бутылке Клейна // Вестник Челябинского гос. ун-та. - 2012. - Т. 280, № 26.

9. Карпухин М.А. Немаксимальность экстремальных метрик на торе и бутылке Клейна // Математический сборник. - 2012. - Т. 280, № 26.

10. Журавлев В.Г. Множества ограниченного остатка на двулистной накрывающей бутылки Клейна // Записки научных семинаров СанктПетербургского отделения математического ин-та им. В.А. Стеклова РАН. - 2014. - Т. 429, № 29.
11. Немировский С.Ю. Гомологический класс лагранжевой бутылки Клейна // Известия Российской академии наук. Серия математическая. 2009. - T. 73 , № 4.

12. Шевчишин В.В. Лагранжевы вложения бутылки Клейна и комбинаторные свойства группы классов отображений // Известия Российской академии наук. Серия математическая. - 2009. T. 73 , № 4 .

13. Козлов И.К. Классификация лагранжевых расслоений // Математический сборник. 2010. - T. 201, № 11 .

14. Шалагинов М.Ю., Иванов М.Г., Долгополов М.В. Задачи с оператором Лапласса на топологических поверхностях // Вестник Самарского гос. техн. ун-та. Серия: Физико-математические науки. - 2011. - № 2 .

15. Борисович Ю.Г., Близняков Н.М., Израилевич Я.А., Фоменко Т.Н. Введение в топологию. - М., 1995.

16. Картан Э. Риманова геометрия в ортогональном репере. - МГУ, 1960.

17. Кобаяси Ш., Номидзу К. Основы дифференциальной геометрии. - Т. 2. - М., 1981.

18. Позняк Ю.Г., Шикин Е.В. Дифференциальная геометрия: первое знакомство. - М., 1990. 